\title{
Uso de la ecografía como una herramienta no invasiva decisiva para determinar el tamaño preciso del tubo endotraqueal en niños anestesiados
} Use of ultrasonography as a noninvasive decisive tool to determine
the accurate endotracheal tube size in anesthetized children

\author{
Prof. asist. Dra. Gulnur Gollu ${ }^{a}$, Dr. A. Onat Bermede ${ }^{b}$, Dr. Farid Khanmammadov, \\ Dr. Ufuk Ates ${ }^{a}$, Dr. Sinan Genc c, Prof. adj. Dra. Ozlem Selvi Can ${ }^{b}$, Prof. Dr. Suat Fitozc, \\ Prof. Dr. Zekeriyya Alanoglu y Prof. Dr. Aydin Yagmurlu
}

\section{RESUMEN}

Introducción. Es difícil determinar el tamaño adecuado y la posición traqueal correcta del tubo endotraqueal (TET) en los niños. El objetivo de este estudio fue determinar el diámetro traqueal en los niños mediante el uso de la técnica ecográfica como herramienta objetiva y compararlo con fórmulas de uso frecuente basadas en la edad para calcular el tamaño del TET.

Pacientes y métodos. Se inscribió de forma prospectiva a pacientes a los que se les iba a practicar una cirugía programada en un hospital pediátrico de alta complejidad. Se determinó el diámetro traqueal transversal infraglótico mediante ecografía. Un anestesista, que no podía ver el examen ecográfico, determinó el tamaño del tubo y realizó la intubación evaluando el espacio entre las cuerdas vocales con la ayuda de la vista directa de un laringoscopio. Se registraron los diámetros traqueales medidos con las ecografías, los diámetrosdelostubos, los controles de presión/ pérdida de aire y los resultados de los cálculos del tamaño de los tubos basados en la edad.

Resultados. Se inscribieron en total 61 pacientes con una media de edad de $12 \pm 4,21(2-17)$ y un peso medio de $38 \pm 22,94$ (10-106). El diámetro de la tráquea en la medición ecográfica fue de 13,0 (11,4-15,1). El diámetro externo del TET determinado por el anestesista fue de $8,42 \pm 1,43$, el calculado por la fórmula de Cole fue de $9,0 \pm 1,42$, el calculado por la fórmula de Khine fue de $7,67 \pm 1,46$ y el calculado por la fórmula de Motoyama fue de 8,33 1,42. En 31 (47,7\%) pacientes, se insufló el manguito después de la colocación del TET debido a la pérdida de aire. El tubo tuvo que reemplazarse por uno más grande a causa de la pérdida excesiva de aire en un paciente. Se halló una correlación intraclase deficienteentre los cálculos del diámetro traqueal determinado por ecografía y los cálculos del diámetro del tubo basado en la edad (diámetro traqueal frente a Cole $[0,273]$, Khine [0,207] y Motoyama [0,230]).

Conclusión. La medición ecográfica del diámetro traqueal transversal es un método adecuado para determinar el tamaño correcto del tubo endotraqueal en comparación con las fórmulas basadas en la edad.

Palabras clave: niño, intubación endotraqueal, tráquea, ecografía. http: / / dx.doi.org/10.5546/ aap.2018.172

Texto completo en inglés:

http: / / dx.doi.org/10.5546/ aap.2018.eng.172

Cómo citar: Gollu G, Bermede AO, Khanmammadov F, et al. Uso de la ecografía como una herramienta no invasiva decisiva para determinar el tamaño preciso del tubo endotraqueal en niños anestesiados. Arch Argent Pediatr 2018;116(3):172-178.

\section{INTRODUCCIÓN}

En los niños, determinar el tamaño correcto del tubo endotraqueal (TET) representa un desafío. ${ }^{1}$ En la rutina diaria, se usan con frecuencia fórmulas relacionadas con la edad para determinar el tamaño correcto del tubo, aunque dichas fórmulas pueden no ser adecuadas para todos los niños. ${ }^{1-3}$ Un TET calculado mediante una fórmula puede ser pequeño y no proporcionar apoyo suficiente del respirador, además de aumentar la resistencia pulmonar, causar una monitorización inadecuada del $\mathrm{CO}_{2^{\prime}}$ aumentar el riesgo de contaminación del quirófano con el agente anestésico y aumentar el riesgo de aspiración. 1,2,4,5 Por otro lado, cuando se colocan tubos endotraqueales más grandes, incluso después de una intubación breve, puede producirse una lesión tipo úlcera en las vías respiratorias altas, isquemia y cicatrización patológica que lleve a la estenosis infraglótica. ${ }^{1,2,4-6} \mathrm{El}$ cálculo adecuado del tamaño correcto del TET debe ser la prioridad principal de los médicos para el manejo satisfactorio de la intubación en el contexto clínico. 
Estudios recientes muestran que la ecografía podría ser una técnica fiable para evaluar el diámetro transversal de la tráquea. Si bien existen diversos estudios en adultos para determinar el tamaño correcto del TET, se necesitan más estudios en la población pediátrica para evaluar la validez clínica de la ecografía en el manejo de la intubación y la correlación con las técnicas de cálculo convencionales. ${ }^{1,2,5}$

El objetivo de este estudio fue determinar el diámetro traqueal en los niños mediante ecografía y su correlación con las fórmulas de cálculo del tamaño del TET basadas en la edad.

\section{PACIENTES Y MÉTODOS}

Este estudio fue aprobado por el Comité de Ética local (Facultad de Medicina de Ankara Üniversitesi, Comité de Ética, director Prof. Dr. Mehmet Melli, número de registro: 09.09.2014, 17-743-14, 27.10.2014) y se realizó de acuerdo con las normas de ética descritas en la versión actual de la Declaración de Helsinki y el consentimiento informado por escrito. Se inscribió en el estudio a pacientes con clasificación del estado físico I-II según la Sociedad Estadounidense de Anestesistas (American Society of Anesthesiologists, ASA) que tenían una cirugía programada con anestesia general entre enero de 2015 y junio de 2015. Los criterios de exclusión fueron cualquier anomalía facial o de las vías respiratorias, falta de consentimiento informado, dificultad anticipada en la intubación y antecedentes de alergia al gel de la ecografía. El mismo anestesista especializado, que no podía ver los resultados del examen ecográfico, realizó todas las intubaciones.

Se medicó previamente a todos los pacientes con $0,5 \mathrm{mg} / \mathrm{kg}$ de midazolan por vía oral. En los pacientes sin acceso vascular, la inducción de la anestesia se logró con sevoflurano al $8 \%$ en una mezcla de $\mathrm{O}_{2} / \mathrm{NO}_{2}$ al $50 \%$. En cuanto se logró el acceso vascular, se administraron 0,6-1,2 $\mathrm{mg} / \mathrm{kg}$ de bromuro de rocuronio. Si el paciente tenía un acceso vascular al momento del ingreso al quirófano, se utilizaron $2-3 \mathrm{mg} / \mathrm{kg}$ de propofol intravenoso y $0,6-1,2 \mathrm{mg} / \mathrm{kg}$ de bromuro de rocuronio para lograr la inducción de la anestesia. El área traqueal infraglótica se midió mediante ecografía (Siemens, Acuson X150, sonda lineal de 13-5 Hz, CA, EE. UU.) en un plano transversal de $90^{\circ}$ cuando la presión en las vías respiratorias era de 0 mbar durante

FIGURA 1. La posición del ecógrafo, el cirujano y el anestesista

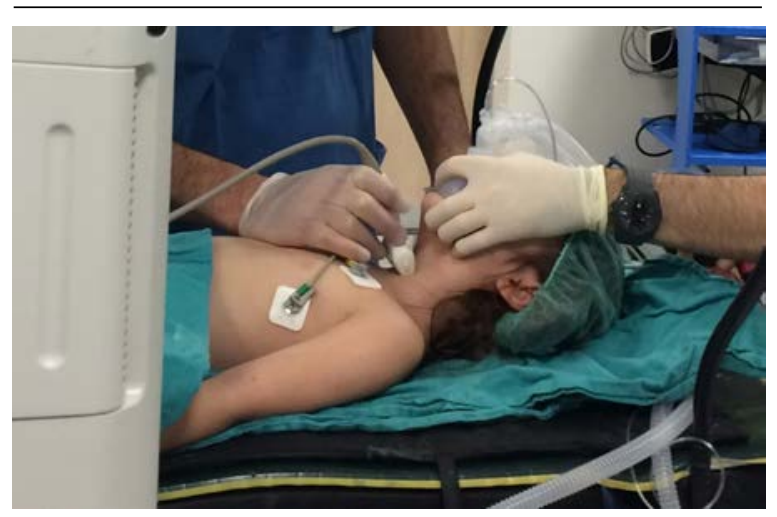

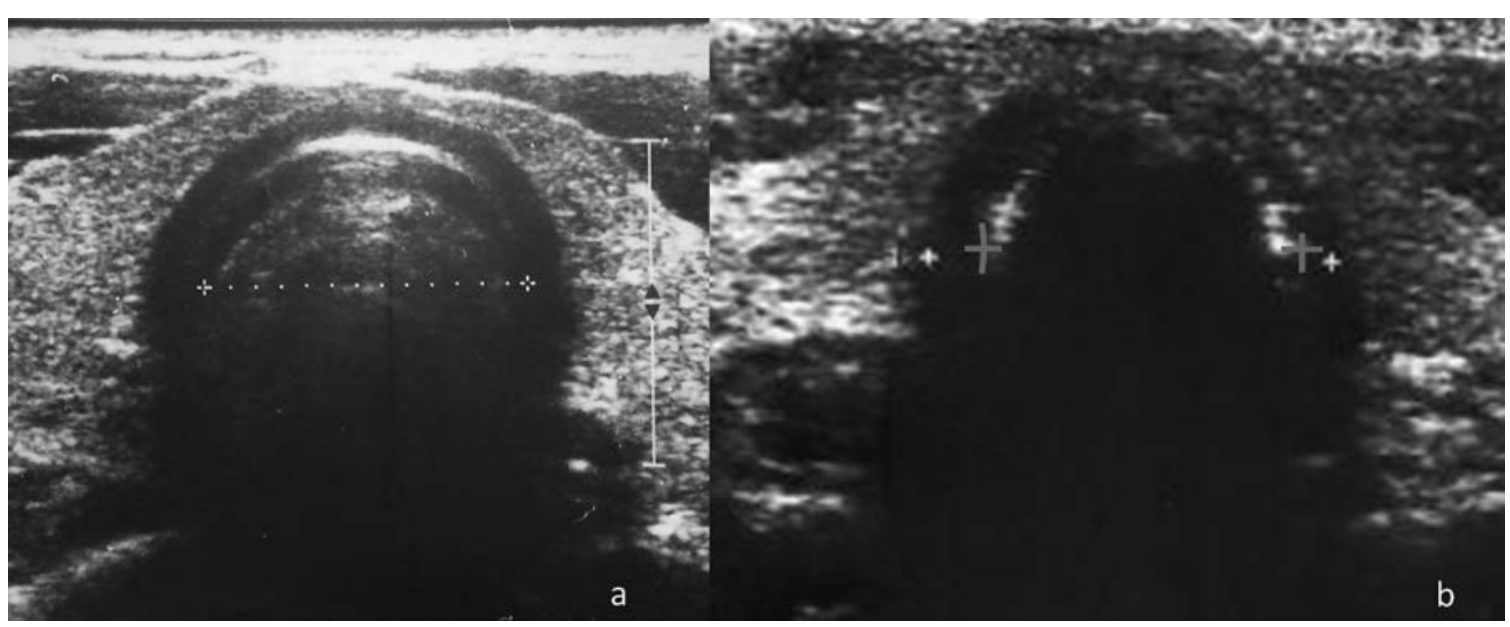

2a. Medición del diámetro traqueal transversal con ecografía.

2b. Control ecográfico de la colocación del TET. 
la ventilación vía mascarilla (en la espiración) y la cabeza estaba en posición neutra (Figura 1). Las mediciones fueron realizadas por el mismo cirujano, quien había recibido una capacitación durante dos semanas sobre la técnica de medición ecográfica del diámetro traqueal por parte de un radiólogo experimentado (Figura 2). El cirujano permaneció del lado derecho del paciente y el ecógrafo se colocó a la izquierda. El monitor del ecógrafo se giró hacia el lado del cirujano, de modo que el anestesista no pudiera verlo (Figura 2a). Se aplicó gel para ecografía en el cuello del paciente, y el transductor se colocó de forma transversal sobre el cuello para medir el área infraglótica inmediatamente inferior a las cuerdas vocales en un plano transversal. El anestesista, que no podía ver las imágenes ni las mediciones del ecógrafo, determinó el tamaño del tubo y realizó la intubación evaluando el espacio entre las cuerdas vocales con la ayuda de la vista directa de un laringoscopio. Se utilizaron tubos con manguito (Kaishou, Jiangsu, China) en todos los procedimientos. La posición intratraqueal del TET se verificó con una ecografía después del procedimiento (Figura $2 b$ ). Como métodos secundarios de verificación, el anestesista determinó el $\mathrm{CO}_{2}$ telespiratorio, los niveles numéricos de $\mathrm{CO}_{2}$ y los ruidos respiratorios bilaterales. Después de la colocación del TET, la válvula de exhalación se configuró en 20$30 \mathrm{cmH}_{2} \mathrm{O}$ y se colocó un estetoscopio en la boca para detectar la pérdida de aire, con la cabeza en posición neutra. El procedimiento se consideró satisfactorio cuando la pérdida no era

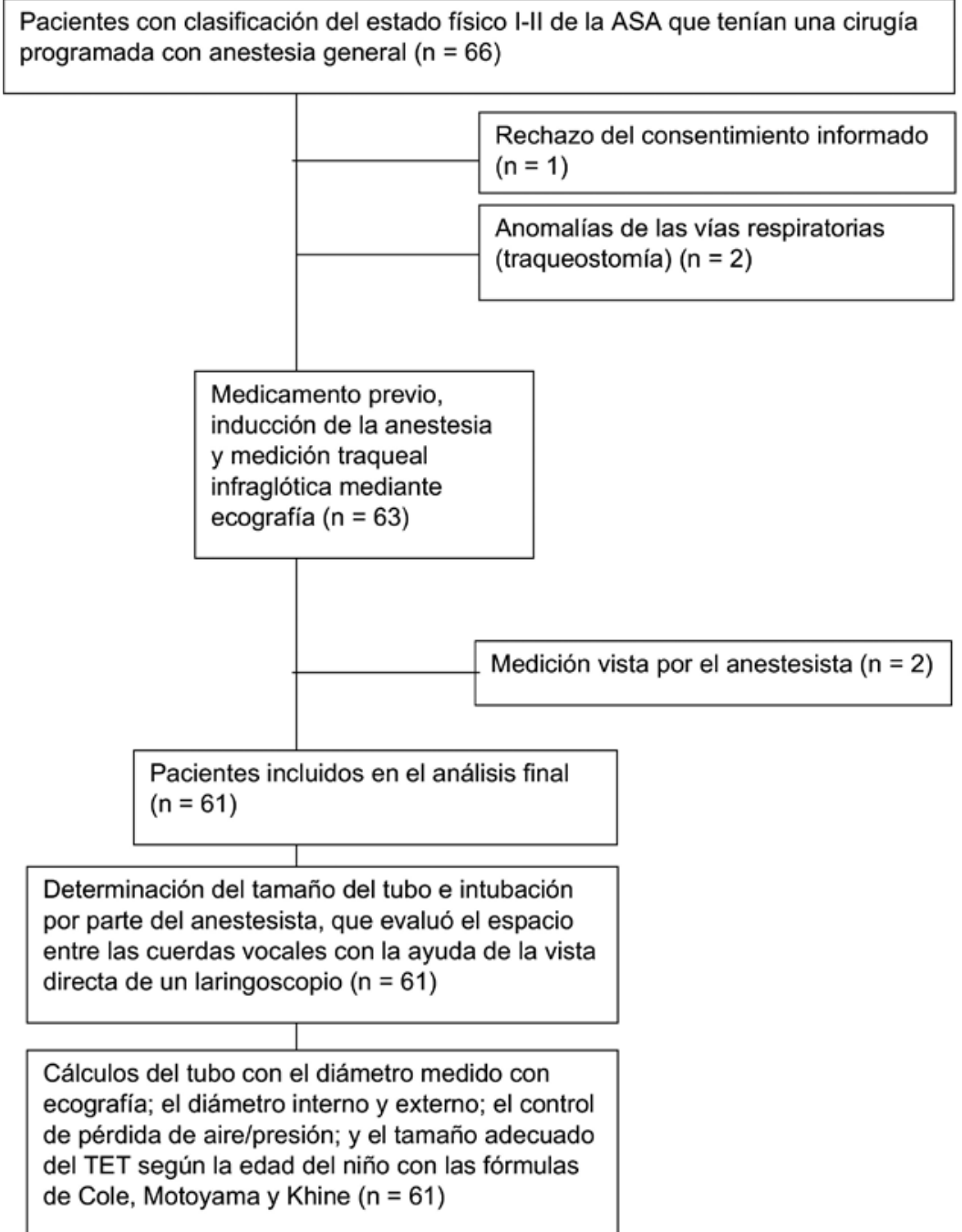


significativa; por el contrario, si no había pérdida de aire en absoluto, esto llevaba al anestesista a pensar que el tubo era demasido grande y entonces lo reemplazaba por uno más pequeño. En el caso de una pérdida excesiva, el manguito del TET se insuflaba con el menor volumen para prevenir la pérdida de aire del manguito. Si con una presión de insuflación de $20-30 \mathrm{cmH}_{2} \mathrm{O}$ la pérdida de aire continuaba, se consideraba que el TET era más pequeño que el tamaño preciso y se aumentaba el tamaño del tubo con manguito en media unidad. Los mismos procedimientos se repitieron cuando se reemplazó el TET. Después del procedimiento, se realizaron cálculos del tubo con el diámetro medido con ecografía; el diámetro interno y externo; el control de pérdida de aire/presión; y el tamaño adecuado del TET según la edad del niño con las fórmulas de Cole (DI $[\mathrm{mm}]=0,25 \times$ [edad en años] +4 ), Motoyama

(DI $[\mathrm{mm}]=0,25 \times$ [edad en años] $+3,5)$ y Khine

(DI $[\mathrm{mm}]=0,25 \times$ [edad en años] + 3), ${ }^{2,7-9}$

\section{Análisis estadístico}

El análisis estadístico se realizó con el paquete estadístico SPSS para Windows 15. Los estadísticos descriptivos incluyeron la

TABla 1. Datos demográficos y tipo de intervenciones quirúrgicas de los pacientes. Mediana (mín.-máx.) y cantidad de pacientes

\begin{tabular}{lc}
\hline Cantidad de pacientes & 61 \\
Edad (años) & $12 \pm 4,21(2-17)$ \\
Sexo (F/M) (n) & $26 / 35$ \\
Peso corporal (kg) & $38 \pm 22,94(10-106)$ \\
Tipo de intervenciones quirúrgicas (n) & 34 \\
$\quad$ Apendicitis aguda & 6 \\
$\quad$ Apendicitis con perforación & 2 \\
Colelitiasis & 2 \\
Quiste de ovario & 17 \\
Otro & \\
Tipo de cirugía (n) & 47 \\
Laparoscópica & 14 \\
Abierta/No laparoscópica & \\
\hline
\end{tabular}

media \pm desviación estándar para las variables con distribución normal, la mediana (mínimomáximo) para las variables sin distribución normal y la cantidad de casos y los porcentajes (\%) para las variables nominales. Las diferencias entre las medias y las medianas se compararon con la prueba t de Student y la prueba U de Mann-Whitney, respectivamente. Las variables nominales se compararon con la prueba de $\chi^{2}$ de Pearson o la prueba exacta de Fisher.

La concordancia entre los dos métodos se calculó mediante el coeficiente de correlación intraclase. Se utilizó el método de Bland Altman para calcular las diferencias entre las mediciones, con los intervalos de confianza del 95\%; los resultados se muestran en un gráfico de Bland Altman. La correlación entre las variables continuas se evaluó mediante el análisis de correlación de Spearman para las variables sin distribución normal y mediante la prueba de correlación de Pearson para las variables de distribución normal. Un valor de $p<0,05$ se consideró estadísticamente significativo.

\section{RESULTADOS}

En la Figura 3, se muestra el diagrama de flujo de los pacientes incluidos. Los datos demográficos y el tipo de intervenciones quirúrgicas de los pacientes inscritos en el estudio se presentan en la Tabla 1. De los niños, cuya edad era de 2 a 17 años (mediana de $12 \pm 4,21$ años), 35 eran varones y 26 eran mujeres. El intervalo de peso de los niños fue de 10-106 kg (mediana de $38 \pm 22,94 \mathrm{~kg}$ ). Los niños recibieron anestesia general por apendicitis aguda $(n=34)$, apendicitis con perforación $(n=6)$, colelitiasis $(n=2)$, quiste de ovario $(n=2)$ y otras indicaciones $(\mathrm{n}=17)$. Se realizó una intervención laparoscópica en 47 (77\%) niños.

El menor y el mayor diámetro interno del TET utilizado para la intubación fue de $4 \mathrm{~mm}$ y $8,5 \mathrm{~mm}$, respectivamente, con una mediana del diámetro interno de 6,5 mm. Después de la colocación del TET, el manguito se insufló

TABla 2. Diámetro de la tráquea medido por ecografía, diámetros externos del tubo endotraqueal determinados por el anestesista y fórmulas basadas en la edad (Cole, Khine y Motoyama)

\begin{tabular}{lcc}
\hline & Mediana, [Percentil (10-90)] & Media \pm DE \\
\hline Diámetro de la tráquea (medido por ecografía) $(\mathrm{mm})$ & $13,0,[11,4-15,1]$ & $13,57 \pm 2,89$ \\
Diámetro externo del tubo endotraqueal $(\mathrm{mm})$ & & $8,42 \pm 1,43$ \\
Determinado por el anestesista & $8,7,[7,3-9,3]$ & $9,0 \pm 1,42$ \\
Calculado por la fórmula de Cole & $9,3,[8,0-10,1]$ & $7,67 \pm 1,46$ \\
Calculado por la fórmula de Khine & $8,0,[6,7-9,0]$ & $8,33 \pm 1,42$ \\
Calculado por la fórmula de Motoyama & $8,7,[7,3-9,4]$ & \\
\hline
\end{tabular}

DE: desviación estándar. 
tras detectar la pérdida de aire en $31(47,7 \%)$ pacientes. El tubo se cambió por uno más grande en un paciente solamente debido a la pérdida excesiva de aire con una presión del manguito de $30 \mathrm{cmH}_{2} \mathrm{O}$. No se observó ninguno de los signos de obstrucción de las vías respiratorias altas en ningún paciente durante la fase de recuperación de la anestesia ni después del alta del hospital. No se observó ningún caso de intubación esofágica accidental.

En la Tabla 2, se muestran el diámetro de la tráquea medido por ecografía, los diámetros externos del tubo endotraqueal determinados por el anestesista y las fórmulas basadas en la edad. Se compararon el diámetro transversal determinado por ecografía y el diámetro del tubo determinado por el anestesista, y se halló un coeficiente de concordancia de 0,273 (valor de F 3,537) $(p=0,001)$ (Figura 4a). El coeficiente de correlación intraclase de los diámetros transversales determinado por la fórmula de Cole y por ecografía fue de 0,273 (valor de F 2,10) ( $p=0,001$ ) (Figura $4 b$ ). Se halló una diferencia significativa entre el diámetro traqueal determinado por ecografía y el determinado por la fórmula de Khine $(p=0,001)$, con un coeficiente de correlación intraclase de 0,207 (valor de F 2,957) (Figura 4c). El coeficiente de correlación intraclase del diámetro transversal determinado por ecografía y el determinado por la fórmula de Motoyama fue de 0,230 (valor de F 2,787) ( $p=0,001)$ (Figura $4 d$ ). No se halló una diferencia significativa entre los diámetros determinados por las fórmulas disponibles y por el anestesista $(p>0,05)$.

FIGURA 4. Coeficiente de correlación intraclase entre los métodos

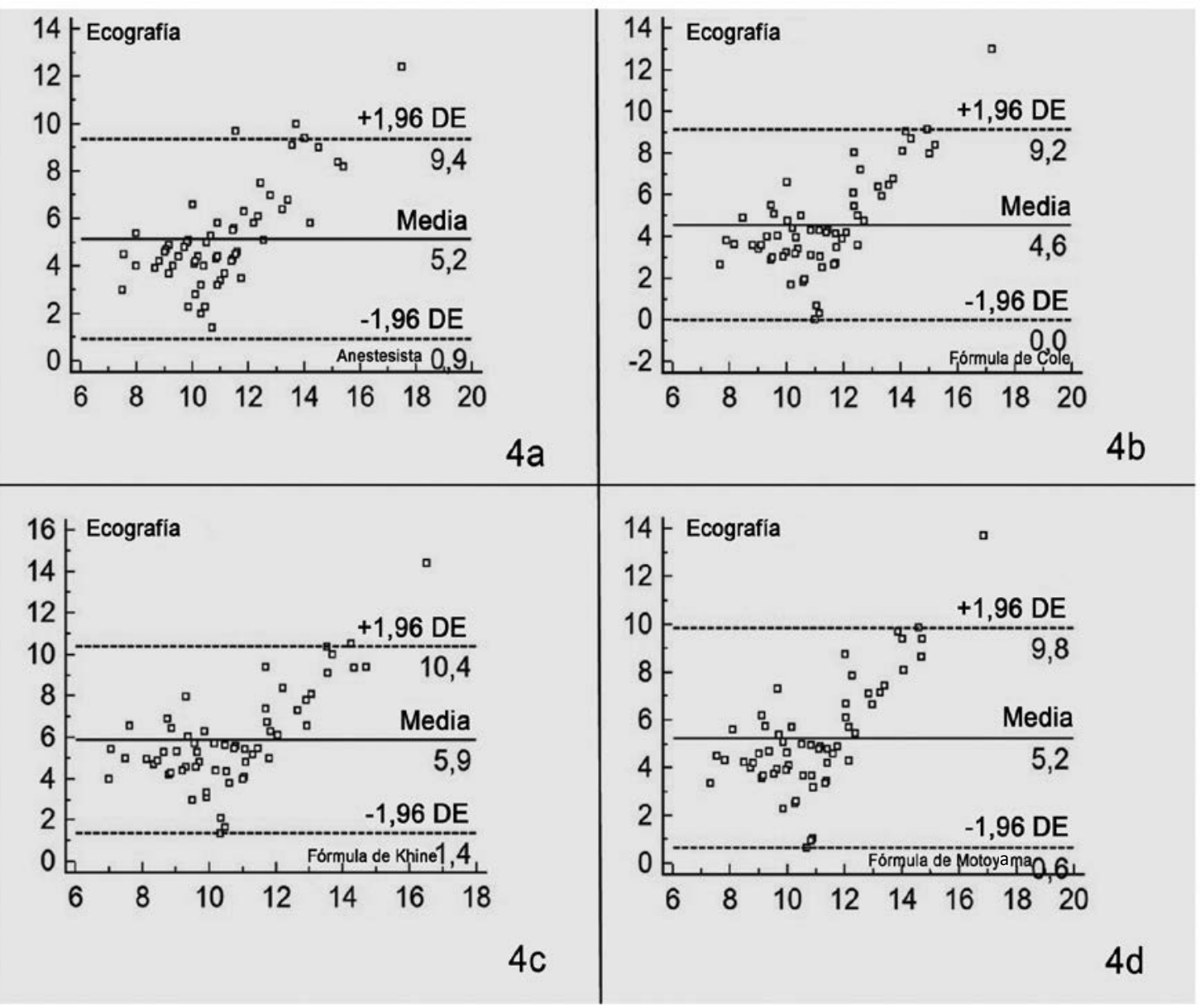

4a: Comparación entre la medición ecográfica del diámetro traqueal transversal y la medición del anestesista. 4b: Comparación entre la medición ecográfica del diámetro traqueal transversal y la fórmula de Cole. 4c: Comparación entre la medición ecográfica del diámetro traqueal transversal y la fórmula de Khine. 4d: Comparación entre la medición ecográfica del diámetro traqueal transversal y la fórmula de Motoyama. 


\section{DISCUSIÓN}

En este estudio, el diámetro traqueal transversal en el área infraglótica determinado por ecografía se compara con el diámetro determinado por un anestesista y por las fórmulas disponibles. Se halló una diferencia significativa entre el diámetro traqueal determinado por ecografía y el diámetro determinado por un anestesista y por las fórmulas disponibles.

En la población de pacientes pediátricos, no siempre es fácil determinar el tamaño adecuado del TET. La variabilidad en el tamaño del tubo puede deberse a la experiencia del operador y a la desproporción en las posibilidades de edad y peso de los niños. Pueden utilizarse fórmulas y métodos para determinar el tamaño del TET y no repetir los procedimientos, a fin de facilitar la intubación..$^{10,11}$ En este estudio, un anestesista con experiencia en el área de anestesiología pediátrica realizó todas las intubaciones guiado por la visualización de las cuerdas vocales. Sin embargo, para un anestesista sin experiencia, podría ser difícil determinar el tamaño del TET con la simple observación del área periglótica. Al parecer, la mayoría de los anestesistas de muchos centros utilizan con frecuencia las fórmulas basadas en la edad, dado que se cree que pueden brindar una solución objetiva a un problema clínico. ${ }^{1,4}$ Además, en este estudio hemos demostrado una diferencia significativa entre el diámetro traqueal medido por ecografía, el diámetro externo del TET determinado por el anestesista y diversas fórmulas basadas en la edad. Esta diferencia clínica exige un método más preciso y organizado para determinar el tamaño exacto del tubo. Los resultados de nuestro estudio indican que la ecografía puede ser una técnica objetiva para que los médicos evalúen el diámetro traqueal, y esta confirmación puede ayudar a que el médico calcule el tamaño correcto y más adecuado del TET para cada paciente individual.

Estudios han demostrado que el diámetro transversal determinado en el área infraglótica es más estrecho que el diámetro anteroposterior; se prefiere medir el diámetro transversal tanto por la conveniencia de la medición como por la estrechez. ${ }^{6}$

La calcificación laríngea comienza después de los 30 años. Debido a que la calcificación laríngea es un hallazgo no previsto en los niños, no hubo limitaciones relacionadas con la calcificación al realizar la medición ecográfica en esta población. ${ }^{6}$
Schramm y col., informaron que la medición ecográfica del diámetro transversal del área infraglótica se asoció con menos repeticiones de los procedimientos de intubación en comparación con lo determinado por las fórmulas basadas en la edad en niños menores de 5 años. ${ }^{1}$ Asimismo, se ha demostrado que la medición ecográfica y las fórmulas basadas en la edad dan como resultado un tamaño correcto del tubo con una tasa del $48 \%$, si bien en otros estudios se informaron tasas del $47 \%$ al $77 \%$. ${ }^{1,2}$ Algunos autores han informado que el diámetro infraglótico puede medirse mediante radiografía de tórax, ecografía y resonancia magnética nuclear. ${ }^{2-4,6}$ Shibasaki y col., al igual que en el estudio actual, demostraron que la ecografía era un buen factor pronóstico en este contexto. $^{2}$

La limitación del estudio actual es que la mayoría de los sujetos operados estuvieron intubados durante períodos breves (media de 40 minutos) y todos los tubos se retiraron después de la cirugía. Por lo tanto, el anestesista puede haber preferido el uso de TET pequeños para evitar el edema posquirúrgico y la obstrucción laríngea. Sin embargo, no se halló una diferencia significativa entre los diámetros determinados por las fórmulas y las mediciones del anestesista. Otra limitación del estudio es que la determinación del diámetro traqueal transversal y la verificación de la posición del tubo entre las cuerdas vocales mediante ecografía requieren un breve período de capacitación. Además, en este estudio, el tamaño adecuado del TET determinado por evaluación ecográfica no fue lo que se utilizó en los pacientes, por lo que no podemos comentar sobre la pérdida de aire o los problemas posquirúrgicos en las vías respiratorias cuando se determina el tamaño del TET mediante evaluación ecográfica.

\section{CONCLUSIÓN}

El uso de la ecografía se asocia con una determinación más adecuada del tamaño del TET, lo que reduce los procedimientos de cambio del tubo en comparación con la determinación realizada por los anestesistas y las fórmulas disponibles. Esta evaluación no invasiva sería beneficiosa para los niños que necesitan una intubación.

La medición ecográfica del diámetro traqueal transversal es un método adecuado para determinar el tamaño correcto del tubo endotraqueal en comparación con las fórmulas basadas en la edad. 


\section{REFERENCIAS}

1. Schramm C, Knop J, Jensen $\mathrm{K}$ y col. Role of ultrasound compared to age-related formulas for uncuffed endotracheal intubation in a pediatric population. Paediatr Anaesth 2012;22(8):781-6.

2. Shibasaki M, Nakajima Y, IshiiS y col. Prediction of pediatric endotracheal tube size by ultrasonography. Anesthesiology 2010;113(4):819-24.

3. Hardee PS, Ng SY, Cashman M. Ultrasound imaging in the preoperative estimation of the size of tracheostomy tube required in specialize doperations in children. $\mathrm{Br} \mathrm{J}$ Oral Maxillofac Surg 2003;41(5):312-6.

4. Gupta K, Gupta PK, Rastogi B y col. Assessment of the subglottic region by ultrasonography for estimation of appropriate size endotracheal tube: A clinical prospective study. Anesth Essays Res 2010;6(2):157-60.

5. Orf J, Thomas SH, Ahmed W y col. Appropriateness of endotracheal tube size and insertion depth in children undergoing air medical transport. Pediatr Emerg Care
2000;16(5):321-7.

6. Lakhal K, Delplace X, Cottier JP y col. The feasibility of ultrasound to assess subglottic diameter. Anesth Analg 2007:104(3):611-4.

7. Khine HH, Corddry DH, Kettrick RG y col. Comparison of cuffed and uncuffed endotracheal tubes in young children during general anesthesia. Anesthesiology 1997;86(3):627-31.

8. Cole F. Pediatric formulas for the anesthesiologist. AMAJ Dis Child 1957;94(6):672-3.

9. Motoyama EK. Endotracheal intubation. En:MotoyamaEK, Davis PJ, eds, Smith's Anesthesia for infants and children. $5^{\text {th }}$ ed. St Louis: Mosby; 1990.Págs.269-75.

10. Chou HC, Tseng WP, Wang $\mathrm{CH}$ y col. Tracheal rapid ultrasound exam (T.R.U.E.) for confirming endotracheal tube placement during emergency intubation. Resuscitation 2011;82(10):1279-84.

11. Göksu E, Sayrac V, Oktay C y col. How to stylet use can effect confirmation of endotracheal tube position using ultrasound. Am J Emerg Med 2010;28(1):32-6.

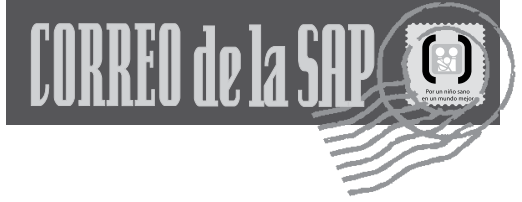

Los siguientes resúmenes y comentarios de trabajos seleccionados se encuentran disponibles en la versión electrónica de este número.

ARCH DIS CHILD. 2017 OCT;102(10):888-893.

Ahogamiento en la infancia: el rol de las enfermedades preexistentes (Franklin $R C$, et al. Drowning fatalities in childhood: the role of pre-existing medical conditions)

Comentario: Prof. Juliana M. Pochetti. Miembro del Comité Nacional de Medicina del Deporte SAP.

\section{PEDIATR PULMONOL. 2018 JAN 12.}

Nebulización con solución salina hipertónica en niños hospitalizados con bronquiolitis moderadamente grave, debida a infección por virus respiratorio sincicial: estudio multicéntrico aleatorizado controlado (Morikawa Y, et al. Nebulized hypertonic saline in infants hospitalized with moderately severe bronchiolitis due to RSV infection: A multicenter randomized controlled trial)

Comentario: Dr. Álvaro Teijeiro. Servicio de Neumonología. Hospital Pediátrico de Córdoba.

\section{PEDIATR PULMONOL. 2018 JAN;53(1):88-94.}

Infección aguda por bocavirus humano 1 confirmada por serología, en la neumonía del niño adquirida en la comunidad (Nascimento-Carvalho AC, et al. Serologically diagnosed acute human bocavirus 1 infection in childhood community-acquired pneumonia)

Comentario: Dra. Laura Moreno y Dra. María P. Adamo. Facultad de Ciencias Médicas. Universidad Nacional de Córdoba.

\section{J ACQUIR IMMUNE DEFIC SYNDR. 2018;77(4):383-392}

Prevención de la transmisión de HIV-1 a través de la lactancia: eficacia y seguridad del tratamiento antirretroviral materno versus la profilaxis con Nevirapina en el niño durante toda la lactancia en madres infectadas con HIV-1 con alto recuento de células CD4 (Impact Promise). Estudio clínico, abierto, aleatorizado (Flynn PM, et al. Prevention of Hiv-1 transmission through breastfeeding: efficacy and safety of maternal antiretroviral therapy versus infant nevirapine prophylaxis for duration of breastfeeding in hiv1-infected women with high Cd4 cell count (Impaact Promise) a randomized, open label, clinical trial)

Comentario: Dra. Susana Rodríguez. Hospital de Pediatría "Prof. Dr. Juan P. Garrahan".

\section{ARCH DIS CHILD FETAL NEONATAL ED 2018; 103:F6-F14.}

Ensayo aleatorizado del clampeo del cordón en prematuros muy pequeños y su estabilización inicial (Duley L, et al. Randomised trial of cord clamping and initial stabilisation at very preterm birth)

Comentario: Dr. José María Ceriani Cernadas. Sociedad Argentina de Pediatría. 